\title{
Production d'autoreformulations autoamorcées par des apprenants adultes du français et capacité de mémoire de travail
}

\author{
Daphnée Simard \\ Université du Québec à Montréal \\ Tatiana Molokopeeva \\ Université du Québec à Montréal \\ Yan Qing Zhang \\ Université du Québec à Montréal
}

\begin{abstract}
Résumé
L'étude rapportée dans cet article avait pour objectif d'examiner la relation entre les autoreformulations autoamorcées, soit les révisions que les locuteurs amorcent et exécutent sur leur propre discours (Salonen et Laakso, 2009, p. 859) et la mémoire de travail, définie comme étant un système à capacité limitée responsable de l'emmagasinage temporaire et de la manipulation de l'information (Baddeley, 2012). Bien que quelques études aient examiné la relation entre les autoreformulations autoamorcées et la mémoire de travail, les résultats obtenus présentent des divergences que des différences d'ordre méthodologique permettent d'expliquer. Nous avons donc voulu jeter un éclairage nouveau sur la relation entre ces deux variables en observant les autoreformulations autoamorcées à l'aide d'une tâche de narration à partir d'images et en ayant recours à une tâche numérique complexe afin de mesurer la mémoire de travail. Trente adultes locuteurs non natifs du français ont participé à l'étude. Les résultats obtenus des analyses factorielles en composantes principales montrent une relation différenciée selon le type d'autoreformulations autoamorcées produit.
\end{abstract}

\begin{abstract}
The objective of the study presented in this article was to examine the relationship between self-initiated self-repairs, the revisions that speakers initiate and perform on their own speech (Salonen \& Laakso, 2009, p. 859) and working memory, defined as limited capacity system responsible for the temporary storage and manipulation of information (Baddeley, 2012). Although a few studies have examined the relationship between self-initiated self-repairs and working memory, the results show discrepancies that can be explained by methodological differences. Therefore, we wanted to shed new light on the relationship between these two variables by observing self-initiated self-repairs using an image-based narrative task and a complex numerical task to measure working memory. Thirty adult non-native speakers of French participated in the study. The results obtained from the principal component factor analyses show a differentiated relationship according to the type of self-triggered self-repair produced.
\end{abstract}




\title{
Production d'autoreformulations autoamorcées par des apprenants adultes du français et capacité de mémoire de travail
}

\author{
Introduction
}

La production orale, qui représente un défi de taille en langue seconde (désormais L2) (Kormos, 2006; Segalowitz, 2010), peut être représentée au moyen du modèle de Levelt (adopté tel quel en L2, p. ex., Arroyo, 2003; Bange et Kern, 1996; Griggs, 2002 ou encore adapté en L2, p. ex., de Bot, 1992; Kormos, 2006; Poulisse, 1997). Selon ce modèle, nous produisons des énoncés en trois grandes phases, à savoir au moyen de la conceptualisation, de la formulation et de l'articulation. Or, des lieux de vulnérabilité se trouvent tout au long de ce processus, de la phase de conceptualisation à la phase d'articulation, en particulier lorsque nous nous exprimons dans notre L2 (Segalowitz, 2010). Il est possible d'observer ces lieux de vulnérabilités au moyen des autoreformulations autoamorcées (ARAA), soit les modifications effectuées par le locuteur lui-même sur son discours. Celles-ci seraient particulièrement intéressantes, car elles offriraient des informations directes sur les mécanismes cognitifs ainsi que sur les processus linguistiques en jeu (Kormos, 1999b).

Les études antérieures sur la production orale en L2 mettent notamment en relief l'effet des caractéristiques cognitives (Kormos, 1999b) sur ces lieux de vulnérabilité. Parmi ces caractéristiques cognitives se trouve la capacité de mémoire de travail dont la relation avec la production d'ARAA n'a été, jusqu'à présent, que peu étudiée (p. ex., Ahmadian, 2015 ; Georgiadou et Roehr-Brackin, 2017; Mojavezi et Ahmadian, 2013). Les résultats des quelques études ayant examiné cette relation s'avèrent différents. Toutefois, il semble que des distinctions sur le plan méthodologique expliquent les écarts observés entre les résultats. Nous avons donc voulu, avec notre étude, jeter un nouvel éclairage sur la relation qui existe entre la mémoire de travail et les ARAA produites en L2.

\section{Modèle de production de la parole de Levelt}

Selon le modèle de Levelt (1989, 1999), la production orale commence par une phase de conceptualisation au cours de laquelle le locuteur passe d'une macroplanification (c.-àd., la conceptualisation de ce qui sera dit, soit l'intention de communication) à une microplanification du discours (c.-à-d., la formulation des idées sous forme propositionnelle qui correspondent le mieux aux intentions de communication). Les processus conceptuels qui ont cours lors de cette phase dans le conceptualisateur entrainent la production d'un message préverbal. Ce message préverbal est par la suite transmis au formulateur. C'est lors de cette deuxième phase - phase de la formulation - que l'élaboration du plan articulatoire, c'est-à-dire la transformation de la forme syntaxique du message en forme phonologique, sera produite. Ce plan articulatoire est, enfin, transmis à l'articulateur pour l'articulation, soit la troisième et dernière phase du modèle.

Levelt $(1989,1999)$ propose l'existence d'un autorégulateur (self-monitor) qui effectuerait des vérifications sur la conformité du message relativement aux intentions de communication de départ ou encore en ce qui a trait à la précision langagière et cela à toutes les étapes de la production, de la conceptualisation du message jusqu'à son articulation. La manifestation observable de cette régulation que le locuteur exerce sur sa propre production lorsque le message a été articulé correspond aux autoreformulations 
(Levelt, 1989 en langue première, désormais L1; Kormos 1999a; 2000a, 2000b; 2006 en L2).

\section{Autoreformulations autoamorcées}

D'abord, les reformulations correspondent à « toute manipulation explicite ou toute négociation des objets linguistiques destinée à éclaircir leur forme ou leur sens " (Griggs, 2002, p. 54). Les reformulations qui sont amorcées et accomplies par le locuteur lui-même, soit les ARAA (voir Schegloff et coll., 1977, pour plus de détails) sont particulièrement intéressantes (par rapport à l'autoreformulation qui est amorcée par l'interlocuteur), car ce sont elles qui témoignent du traitement cognitif exécuté par l'apprenant à l'égard de sa propre production orale (p. ex., Arroyo, 2003; Camps, 2003; Fathman, 1980; Gilabert, 2007; Griggs, 1998, 2002, 2007; Kormos, 2000a, 2000b; Lennon, 1984, 1990; O'Connor, 1988; Verhoeven, 1989). ${ }^{1}$ de manière plus précise, elles permettent d'observer les mécanismes cognitifs, psychologiques et linguistiques en jeu lors de la production langagière (Kormos, 1999b, 2006), car elles «signalent une 'activité cognitive de contrôle des énoncés' et démontrent qu'il y a non seulement une réflexion sur la forme de l'énoncé, mais aussi une 'activité métalinguistique' de la part du locuteur» (Royer, 2002, p. 72).

Selon Levelt (1983), les ARAA sont constituées d'un reparandum, soit l'élément étant l'objet d'une modification dans le discours du locuteur, d'une phase de régulation et d'un reparatum, soit la nouvelle formulation.

\section{Figure 1}

Séquence d'ARAA selon Levelt (1983)

\section{moment d'interruption}

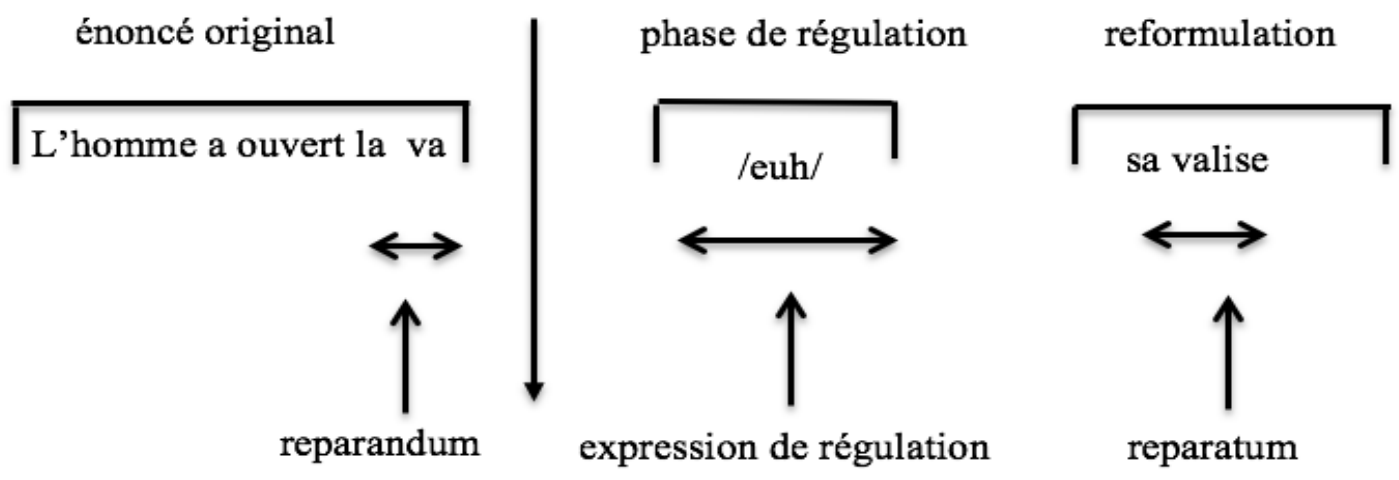

Note. adapté de Levelt (1983) p. 45

Dans la séquence suivante « [...] l'homme a ouvert la va /euh/ sa valise $[\ldots] »$, le reparandum correspond à « la va » et le reparatum à « sa valise », séparés par une phase de régulation représentée par/euh/. Il est à noter que la phase de régulation n'est pas toujours présente dans la structure des ARAA. 
Enfin, il semble que les ARAA issues d'une régulation au niveau du conceptualisateur viseraient un choix lié au discours, tandis que celles issues d'une régulation au niveau du formulateur viseraient la forme de la langue (Zuniga, 2015; Zuniga et Simard, 2019). Ainsi, les types d'ARAA varieraient selon la phase de production orale au cours de laquelle elles ont lieu.

\section{Mémoire de travail et production orale en L2}

Un ensemble de caractéristiques individuelles cognitives interviennent dans la production orale en L2, parmi ces caractéristiques se trouvent la mémoire de travail, soit le système à capacité limitée responsable du stockage temporaire et de la manipulation d'informations (Baddeley, 2012). Elle aurait « [...] pour fonction de maintenir temporairement une petite quantité d'information, sous un format facilement accessible, pendant la réalisation de tâches cognitives diverses (de raisonnement, de compréhension, de résolution de problème, etc.) » (Van der Linden et Colette, 2002, p. 41).

La mémoire de travail consiste en un administrateur central amodal (c.-à-d., sans modalité visuelle, sonore, etc.) à capacité limitée dont les sous-systèmes permettent le maintien temporaire de l'information (Baddeley, 1986, 1992; Baddeley et Hitch, 1974). Cet administrateur central (central executive) a pour fonction de coordonner le passage des informations des sous-systèmes à la mémoire à long terme, soit la boucle phonologique, le calepin visuospatial et le tampon épisodique.

D'abord, la boucle phonologique, considérée comme étant le 'dispositif d'acquisition du langage' (Baddeley et coll., 1998), se compose de l'emmagasinage verbal et de la récapitulation articulatoire. La boucle phonologique serait responsable de la mémoire phonologique, à savoir « la capacité à reconnaitre et à se souvenir de séquences phonologiques dans leur ordre d'apparition » (O'Brien et coll., 2007, p. 558; notre traduction de : " the ability to recognize and remember phonological elements and their order of occurrence »). Celle-ci favoriserait l'acquisition du langage, et subséquemment, des langues, car elle permettrait le maintien actif de nouvelles traces phonologiques temporaire jusqu'à ce que se forment des représentations plus solides (p. 559). Il est à noter que bien que la mémoire phonologique constitue une sous-partie de la mémoire de travail dans le modèle de Baddeley, les deux construits sont toutefois traités comme étant des phénomènes distincts dans la recherche en L2 (p. ex., Harrington et Sawyer, 1992; Martin et Ellis, 2012; Miyake et Friedman, 1998; Wen, 2016). ${ }^{2}$ Ainsi, les chercheurs examineront, d'une part, l'administrateur central de la mémoire de travail, soit l'aspect 'exécutif', en ayant recours à des tâches exigeant à la fois l'emmagasinage et la manipulation de l'information, et d'autre part, ils examineront l'aspect 'emmagasinage à court terme de l'information verbale' afin de mesurer la mémoire phonologique (boucle phonologique). Le calepin visuospatial est pour sa part responsable de l'emmagasinage à court terme de l'information visuospatiale. Enfin, le tampon épisodique est responsable de l'emmagasinage à court terme de l'information qui provient des deux autres sous-systèmes et de la création de représentations multimodales. Il permet également l'interaction entre les sous-systèmes et la mémoire à long terme (Baddeley, 2000, 2010).

À notre connaissance, seules trois études ont mis en relation l'aspect exécutif de la mémoire de travail et la production d'ARRA en L2. Il s'agit de Mojavezi et Ahmadian (2013), d'Ahmadian (2015), et de Georgiadou et Roehr-Brackin (2017). 
D'abord, Mojavezi et Ahmadian (2013) ont examiné le lien entre une mesure de l'aspect exécutif de la mémoire de travail et la production d'ARAA en L1 et en L2 chez 40 locuteurs du farsi apprenants de l'anglais L2 (13 hommes et 27 femmes) en ayant recours à une tâche d'empan d'écoute ${ }^{3}$, considérée comme mettant en jeu à la fois l'emmagasinage et le traitement de l'information. Au cours de cette tâche, les participants doivent effectuer un jugement d'acceptabilité sur des phrases entendues dans leur L1 et de mémoriser le dernier mot de chacune d'entre elles. Ils doivent, à la suite de l'écoute des phrases, écrire les mots retenus sur une feuille. Les ARAA ont, quant à elles, été recueillies au moyen d'une tâche de narration basée sur le visionnement d'un dessin animé muet. Les auteurs ont extrait aléatoirement 20 reformulations de chacune des trois minutes de productions transcrites, au début, au milieu et à la fin des narrations. Les ARAA identifiées dans les narrations ont été codées à l'aide d'une tâche de rappel suscité (stimulated recall) ${ }^{4}$ selon qu'elles visaient un changement dans le discours, une modification afin de rendre leur discours plus précis ou encore la correction d'une erreur.

Les résultats obtenus mettent en lumière, dans un premier temps, que les participants de niveau intermédiaire en anglais L2 ont produit plus d'ARAA visant des corrections de la forme que celles visant le discours ou encore celles visant l'ajout de précision. Dans un second temps, les résultats révèlent qu'une meilleure capacité de mémoire de travail est associée de façon statistiquement significative à plus d'ARAA visant des erreurs de formes et à moins d'ARAA visant des changements dans le discours lors de la production en L2.

Quelques années plus tard, Ahmadian (2015) reprend l'étude de Mojavezi et Ahmadian (2013), cette fois en vérifiant si l'aspect exécutif de la mémoire de travail est en relation avec les ARAA lorsque la planification de la production orale est réalisée en temps réel (on-line) (c.-à-d., non préalablement à la tâche). Les participants n'étaient pas limités dans le temps pour effectuer leur production orale. Les 53 participants de l'étude (23 hommes et 30 femmes), locuteurs du farsi et tous de niveau intermédiaire en anglais L2 ont réalisé une tâche d'empan d'écoute qui visait la mesure de la mémoire de travail (la même que celle utilisée par Mojavezi et Ahmadian, 2013), une tâche de narration à partir du visionnement d'un dessin animé et une tâche de rappel suscité, afin d'obtenir davantage de précision relativement aux ARAA produites. Les ARAA ont été codées de la même façon que dans l'étude précédente. Les résultats des analyses de données ont révélé, comme dans l'étude de Mojavezi et Ahmadian (2013), une corrélation positive significative entre la mémoire de travail et les ARAA visant la correction d'une erreur dans la forme et une corrélation négative significative avec les ARAA visant un changement dans le discours.

Georgiadou et Roehr-Brackin (2017) ont, pour leur part, examiné les relations entre l'aspect exécutif de la mémoire de travail mesuré au moyen d'une tâche d'empan de chiffres inversés (Wechsler, 1997) et d'une tâche d'empan d'écoute et les ARAA chez 77 locutrices de l'arabe apprenantes de l'anglais L2 de niveaux débutant et intermédiaire bas. La tâche d'empan de chiffres inversés a été réalisée dans la L1 des participantes, tandis que la tâche d'empan d'écoute a, pour sa part, été effectuée dans leur L2, soit l'anglais. Pour cette tâche, des phrases étaient présentées une à la fois. Après la présentation de chaque phrase, les participantes devaient juger de leur acceptabilité et mémoriser leur dernier mot. Le rappel des mots était effectué à la fin de chaque série de phrases. Afin d'obtenir les ARAA, les participantes ont été soumises à une tâche d'entrevue basée sur l'une des parties de l'International English Language Testing System. Dans la partie utilisée, les participantes devaient parler pendant deux minutes de leur meilleur ami. Une minute de 
préparation leur avait préalablement été accordée. Les auteurs ont codé le nombre d'ARAA produites pendant deux minutes de narration et les ont par la suite codées selon qu'elles visaient un changement dans le discours (c.-à-d., le remplacement d'une information par une autre), une modification afin de rendre le discours approprié (c.-à-d., plus détaillé, précis ou encore moins ambigu), une correction d'une erreur, ou encore une reformulation de leur message (c.-à-d., un changement dans la forme dû à un doute sur la justesse de l'information).

Aucune relation entre les résultats obtenus de la tâche d'empan de chiffres inversés ou encore de la tâche d'empan d'écoute et la production d'ARAA n'a été observée.

\section{Synthèse et question de recherche}

Bien que chacune de ces trois études porte sur la relation entre la production d'ARAA en anglais L2 et l'aspect exécutif de la mémoire de travail mesurée au moyen d'une tâche d'empan d'écoute, elles présentent néanmoins des distinctions quant à leurs éléments méthodologiques respectifs, distinctions qui permettent certainement d'expliquer les écarts observés dans les résultats obtenus. À cet égard, rappelons que bien que Mojavezi et Ahmadian (2013) et Ahmadian (2015) ont observé une relation entre la mémoire de travail et la production d'ARAA, Georgiadou et Roehr-Brackin (2017) n'en ont, quant à elles, observé aucune.

D'abord, les différences dans les résultats peuvent certainement en partie être expliquées par la langue de passation de la tâche d'empan d'écoute. En effet, dans les études de Mojavezi et Ahmadian (2013) et d'Ahmadian (2015), elle a été réalisée dans la L1 des participants, soit le farsi, tandis que dans l'étude de Georgiadou et Roehr-Brackin (2017), les participantes ont effectué la tâche d'empan d'écoute dans leur L2, soit l'anglais. Certains chercheurs ont évoqué le risque d'un effet de chevauchement entre les contenus manipulés dans les études sur la mémoire de travail (p. ex., Turner et Engle, 1989). Ces chevauchements dans les contenus mèneraient à l'observation de relations entre les variables qui ne devraient pas être interprétées d'une manière ou d'une autre.

Selon ces chercheurs, comme la mémoire de travail ne serait pas spécifique à un domaine particulier, mais représenterait plutôt une capacité générale, ses effets seraient observables à travers différents contenus. Ils ont donc proposé des tâches numériques afin de mesurer l'aspect exécutif de la mémoire de travail en invoquant sa qualité générale. Parmi ces tâches numériques se trouvent la tâche d'empan de chiffres inversés que Georgiadou et Roehr-Brackin (2017) ont également utilisée, en plus de leur tâche d'empan d'écoute, et celles impliquant des calculs mentaux ou encore l'identification du plus grand nombre au sein de groupes de nombres (p. ex., Oakhill et coll., 2011). En général, ce type de tâches est effectué dans la langue maternelle des participants afin de contrôler le biais de connaissance de la langue qui est susceptible d'influencer les performances des participants. ${ }^{6}$

Il est à noter que la demande sur les ressources cognitives de la partie visant le traitement de l'information varie d'une tâche numérique à l'autre. Il semble, en effet, que la tâche d'empan de chiffres inversés, comme celle utilisée par Georgiadou et Roehr-Brackin (2017), bien que demandant une bonne capacité d'emmagasinage à court terme, n'impose que peu de demande sur la partie traitement de l'information et ne permet donc pas de mesurer complètement la capacité de « manipulation de l'information » de l'aspect exécutif 
de la mémoire de travail, en particulier, chez une population adulte (p. ex., Colom et coll., 2005; Rosen et Engle, 1997; St Clair-Thompson, 2010). On peut donc penser, d'une part, que la tâche d'empan de chiffres inversés utilisée par Georgiadou et Roehr-Brackin (2017) n'a pas permis de mesurer l'aspect exécutif de la mémoire de travail, soit la manipulation de l'information, et d'autre part, que l'aspect emmagasinage à court terme, la partie la plus présente de la tâche qu'elles ont utilisée, ne soit pas déterminante dans la production d'ARAA.

Il est également possible que les différences observées relèvent de la manière dont les ARAA ont été collectées dans chacune des trois études. En effet, Mojavezi et Ahmadian (2013) et Ahmadian (2015), qui ont obtenu des résultats similaires, ont sélectionné aléatoirement 20 reformulations de chacune des trois minutes de productions transcrites, au début, au milieu et à la fin des narrations basées sur un film muet, tandis que Georgiadou et Roehr-Brackin (2017) ont plutôt travaillé à partir des données brutes d'ARRA, soit toutes les ARAA produites durant deux minutes de production orale, sans les mettre en relation avec le nombre de mots produits par minute, autrement dit sans calcul de ratio (p. ex. Griggs, 1997). Il est à noter que lors d'analyses effectuées sur des fréquences d'éléments langagiers tirés de productions provenant de différents locuteurs, les données obtenues de chaque participant devraient être normalisées en divisant le nombre d'occurrences de l'élément (fréquences) par le nombre de mots produits dans un temps donné comparable entre toutes les productions (voir entre autres Biber et coll., 2006, p. 263). Cette façon de procéder permet de contrôler le fait que certains participants produisent plus de mots dans un temps donné que d'autres participants, augmentant ainsi leur chance de produire davantage d'éléments visés que les participants produisant moins de mots dans le même temps donné de production.

Ainsi il serait intéressant d'examiner la relation entre l'aspect exécutif de la mémoire de travail et les ARAA au moyen d'une tâche impliquant minimalement le traitement verbal et exigeant à la fois l'emmagasinage et le traitement de l'information. Nous avons donc voulu vérifier la relation qu'entretient l'aspect exécutif de la mémoire de travail avec la production d'ARAA en répondant à la question suivante :

Quelle est la relation entre l'aspect exécutif de la mémoire de travail mesurée à l'aide de la tâche du plus grand nombre (Oakhill et coll., 2011) et la production d'ARAA réalisées lors d'une tâche de narration à partir d'images ?

\section{Méthode}

Une étude transversale ayant comme variable dépendante les ARAA manifestes produites lors de la production orale en L2, et comme variable indépendante, la capacité de mémoire de travail des participants a été mise en place.

\section{Participants}

Au total, 30 participants, tous nés en Chine et dont la L1 est le mandarin, ont été recrutés dans la grande région de Montréal. De nos 30 participants, 25 parlaient également l'anglais et un parlait le coréen. Aussi, parmi ces 30 participants, 21 étaient des femmes et 9 étaient des hommes $(M=35,8$; tranche d'âge $=18-50)$. De plus, 27 de nos participants avaient obtenu un baccalauréat en Chine avant d'arriver au Canada et 18 poursuivaient 
leurs études dans un collège ou une université francophone au moment de la collecte des données. Les autres participants travaillent dans un environnement francophone. La durée moyenne de résidence au Québec était de 6,7 ans ( $\min .=3$ mois ; max. $=15$ ans).

\section{Instruments de mesure}

Dans le cadre de notre collecte de données, nous avons eu recours à trois instruments de mesure, soit une tâche de narration à partir d'images, une tâche de mémoire de travail et un questionnaire de données sociodémographiques. Nous les détaillons dans ce qui suit.

\section{Tâche de production orale}

Nous nous sommes servies d'une tâche de narration à partir d'images pour obtenir des données relatives au niveau de compétence langagière de nos participants et à leur production d'ARAA en L2. Cette tâche a souvent été utilisée dans les études sur la production orale en général (Munro et Derwing, 1995; Rossiter et coll., 2008) et dans celles sur les ARAA, en particulier (p. ex., Bange et Kern, 1996; Camps, 2003, Gilabert, 2007; Simard et coll., 2011; Simard et coll., 2016; Zuniga, 2015; Zuniga et Simard, 2019). Elle est considérée comme favorisant la production d'ARAA (p. ex., Gilabert, 2007).

Pour la tâche, 47 images de la bande dessinée Tintin au Congo (Hergé, 1931, pp. 5-8) ont été retenues. Elles ont été reproduites et collées sur des cartons afin que les participants puissent avoir une vue globale des événements. Comme c'est souvent le cas dans ce genre d'étude, du temps de préparation a été accordé à nos participants afin de favoriser la complexité grammaticale lors de la production de narrations (Yuan et Ellis, 2003; Foster et Skehan, 1996). Un niveau plus élevé de complexité mènerait à la production d'un plus grand nombre d'ARAA (Gilabert, 2007). Les participants ont reçu la consigne de regarder les images et s'ils le souhaitaient de prendre des notes sur une feuille afin de les aider à planifier leur narration (Mehnert, 1998). Ils ont toutefois été informés qu'ils n'auraient pas accès à leurs notes au moment d'effectuer leur narration (Tavakoli et Foster, 2008). Environ deux minutes leur ont été accordées préalablement à leur production orale pour planifier leur narration. Enfin, nous n'avons pas imposé de limite de temps lors de la réalisation de la tâche de narration afin de permettre aux participants d'effectuer une planification en temps réel, car cette façon de faire permettrait d'observer un plus grand nombre d'ARAA (Yuan et Ellis, 2003, p. 17).

\section{Mémoire de travail}

La capacité de mémoire de travail a été mesurée d'une tâche nécessitant le traitement simultané d'informations numériques, à savoir la tâche du nombre le plus grand (Oakhill et coll., 2011). Chaque participant voit et entend des séries de trois nombres présentés sur un écran d'ordinateur. Tous les nombres qu'il voit à l'écran sont compris entre 1 et 19 et chaque série contient un nombre inférieur à 10 et deux entre 10 et 19 . Après la présentation d'une série de trois nombres, le participant doit identifier à voix haute le nombre le plus élevé et le mémoriser. Lorsqu'un point d'interrogation apparaît à l'écran, le participant doit alors dire à voix haute tous les nombres les plus grands dans l'ordre dans lequel ils sont apparus à l'écran. 
La tâche est constituée de trois groupes de pratique et de 12 groupes principaux (items cibles). Le premier groupe de pratique contient deux séries de trois nombres (1x2), le deuxième en contient trois séries de trois nombres $(1 \times 3)$ et le dernier, quatre séries de trois nombres $(1 \times 4)$. Les 12 groupes principaux se déclinent comme suit : trois groupes contenant deux séries de trois nombres $(3 \times 2)$, trois groupes contenant trois séries de trois nombres $(3 \times 3)$, trois groupes contenant quatre séries de trois nombres $(3 \times 4)$ et trois groupes principaux contenant cinq séries de trois nombres $(3 \times 5)$. Voici dans la Figure 2 un exemple de trois groupes principaux contenant trois séries de nombres suivis d'un point d'interrogation (tâche du plus grand nombre, Oakhill et coll., 2011).

\section{Figure 2}

Exemple de groupes prinicpaux dans la tâche de mémoire de travail

\begin{tabular}{|c|c|c|}
\hline 4 & 18 & 11 \\
\hline 6 & 12 & 14 \\
\hline 12 & 7 & 16 \\
\hline \multicolumn{3}{|c|}{$?$} \\
\hline
\end{tabular}

Dans ce cas précis, afin de donner la bonne réponse, les participants auraient dû dire à haute voix, en voyant le point d'interrogation à l'écran : 18,14 et 16, c'est-à-dire les nombres les plus élevés dans l'ordre dans lequel ils sont apparus.

Cette tâche a été utilisée pour minimiser les éventuels biais liés au chevauchement des contenus rencontrés dans des tâches telles que celle d'empan de lecture. Elle a été passée dans la L1 des participants, le mandarin, afin d'éviter un éventuel biais de connaissance de la langue, susceptible d'influencer les performances des participants.

\section{Questionnaire sociodémographique}

Enfin, afin d'obtenir des informations générales sur l'âge, le sexe, la durée de résidence au Canada, l'éducation, la L1 et d'autres langues parlées, ainsi que sur l'expérience générale en français des participants, un questionnaire sociodémographique a été passé. Les informations recueillies à partir du questionnaire ont été utilisées pour décrire nos participants ci-dessus.

\section{Procédure}

Toutes les tâches ont été présentées individuellement aux participants. Après avoir signé le formulaire de consentement, ils ont réalisé la tâche de mémoire de travail (Oakhill et coll., 2011) dans leur L1, le mandarin. Ensuite, les participants ont effectué la tâche de narration à partir d'images. Aucun échange verbal entre le participant et l'expérimentateur n'a été autorisé pendant cette tâche. Les narrations ont été enregistrées avec un enregistreur numérique Olympus LS-P2 linéaire. À la fin de la session de test, tous les participants ont rempli le questionnaire sociodémographique. Le tout s'est réalisé en environ 30 minutes.

\section{Traitement des données}

Revue canadienne de linguistique appliquée : 24, 1 (2021) : 138-158 
Dans ce qui suit, nous présentons, d'abord, la procédure utilisée pour la préparation des productions orales. Nous présentons, ensuite, les procédures de codification utilisées pour les ARAA, la compétence langagière et des données obtenues de la tâche de mémoire de travail.

\section{Préparation des narrations}

Les participants ont produit des narrations d'une durée moyenne de 5,53 minutes. Un temps déterminé de production orale, correspondant au dénominateur commun de temps de narration produit par l'ensemble des participants, a été analysé afin de s'assurer de les rendre comparables sur le plan du temps de production (Biber et coll., 2006). Pour ce faire, nous avons déterminé le temps maximum commun pour les narrations de nos trente participants après avoir éliminé les 20 premières secondes, considérées comme étant un échauffement, et après avoir élagué toutes les pauses de plus de deux secondes (Lennon, 1990). Nous avons donc identifié le participant dont la narration était la plus courte parmi nos 30 participants. Cette narration était de 2 minutes 34 secondes, après avoir retiré les 20 premières secondes d'échauffement. Le temps commun maximum de deux minutes et 34 secondes est donc devenu le dénominateur commun de toutes les narrations produites. Tout temps de production supplémentaire a été supprimé des enregistrements. Les narrations ont ensuite été transcrites par deux chercheurs qui ont à tour de rôle vérifié leurs transcriptions. En cas de désaccord, un troisième chercheur s'est chargé de valider la transcription.

\section{Codification $\operatorname{des} \boldsymbol{A R A} A$}

Afin d'identifier les ARAA, nous avons utilisé la structure de séquence d'ARAA proposée par Levelt (1983, p. 45). La codification des ARAA réalisée en parallèle et à l'aveugle par deux juges a mené à un accord s'élevant à $95 \%$. En ce qui concerne la codification des ARAA, nous nous sommes basées sur la procédure proposée par Simard et ses collaborateurs (2011). Les auteurs signalent que les catégories proposées par les chercheurs (p. ex., Bange et Kern, 1996; Fincher, 2006; Kormos, 2000a, 2000b) « varient d'une étude à l'autre, et souvent, ne sont pas opérationnalisées exactement de la même façon, rendant difficiles les comparaisons directes » (p. 424). Simard et ses collaborateurs (2011) ont mis en lumière que le dénominateur commun des nombreuses catégorisations proposées dans ces études en L2, toutes basées sur les travaux de Levelt (1983, 1989), correspond, d'une part, aux modifications liées à la forme (A-forme) et, d'autre part, aux modifications liées au choix de mot ou de groupes de mots (A-choix). Les $A$-forme correspondent aux ARAA dont l'objet est un changement lié au nombre et aux conjugaisons, tandis que les A-choix, correspondent aux ARAA dont l'objet vise un changement d'élément langagier (p. ex., choix de mots, choix de groupes de mots). Dans l'extrait suivant « [...] puis la police dans le bateau // a sauvé // euh la chien // le chien //», la reformulation de « la chien » en « le chien » correspond à une ARAA de type A-forme, tandis que dans cet extrait : « et puis il entre dans le::: boyau // dans le tuyau // l'ARAA est de type A-choix. Deux juges ont codé à l'aveugle et en parallèle les séquences d'ARAA préalablement identifiées selon qu'elles portaient sur la forme (A-forme) ou encore sur le choix (A-choix) d'un élément langagier. Dans les cas de désaccord, un troisième juge a 
tranché. L'accord interjuge calculé à l'aide d'un alpha de Cronbach a révélé un coefficient de 0,84 , considéré comme étant très acceptable (Larson-Hall, 2010).

Enfin, le ratio de reformulations par nombre de mots produits pendant les deux minutes 34 secondes de narration transcrites pour chaque participant a été calculé selon la méthode proposée par Griggs (1997), soit en divisant le nombre de mots produits par le nombre d'ARAA pris globalement d'abord et par type ensuite. Afin de calculer le nombre de mots produits, les secondes parties de répétitions, les faux départs et les mots répétés et reformulés dans les séquences de reformulations sont enlevés et ainsi seuls les mots apportant une information nouvelle sont calculés (p. 410).

\section{Codification de la compétence langagière}

Afin de coder la compétence langagière de nos participants, les narrations qu'ils ont produites ont été codées à l'aide du logigramme élaboré par Turner et Upshur (2002, mais voir aussi Upshur et Turner, 1995, 1999) et adapté par White et Turner (2005) et par Simard et ses collaborateurs (2011). Le logigramme consiste en « un ensemble ordonné de questions binaires relatives aux limites entre les niveaux de la performance évaluée » (Upshur et Turner, 1995, p. 3). Plus précisément, les juges doivent répondre à trois questions conduisant à une note maximale possible de six. Les trois questions visent la cohérence et la cohésion du discours, la précision grammaticale et la richesse lexicale. Ainsi, comme la procédure le demande, trois juges ont écouté l'ensemble des productions orales et ont assigné un résultat allant de 1 à 6 et les résultats accordés par chaque juge ont été additionnés, pour un résultat maximal de 18.

Avant la codification des narrations, les trois juges ont été formés avec différents enregistrements réalisés auprès d'apprenants du français locuteurs natifs du mandarin et possédant différents niveaux de maîtrise de la langue. Le coefficient de fiabilité interjuge pour la codification du niveau de compétence est de ,977 (coefficient d'intercorrélations).

\section{Codification de la tâche de mémoire de travail}

La procédure d'Oakhill (Oakhill et coll., 2011) a été utilisée pour coder les données obtenues à partir de la tâche de mémoire de travail. Selon cette procédure de notation « tout ou rien », un point était attribué à chaque réponse correcte (max. $=15)$.

\section{Analyse des données}

Dans un premier temps, nous avons calculé les moyennes et écarts types pour les résultats obtenus à chacune des tâches. Dans un second temps, nous avons vérifié l'existence de relations entre nos différentes variables. Pour ce faire, nous avons effectué une analyse factorielle en composantes principales. L'analyse en composantes principales permet de rendre compte de la présence d'interrelations entre des variables afin de les regrouper en facteurs (Stafford et Bodson, 2006, p. 58). Ces regroupements de facteurs sont représentés sous forme d'axes (ou 'dimensions') qui révèlent une plus grande variation à l'intérieur des données. Le nombre de dimensions existant à l'intérieur des données est déterminé au moyen du critère de Cattell (1966). Selon ce critère, l'extraction de 
dimensions est effectuée sur la base de la représentation graphique du test d'accumulation de la variation et s'arrête à l'endroit où le plus grand changement dans la pente se produit. L'intervalle de confiance a été établi à 95\%.

\section{Résultats}

Dans ce qui suit, nous présentons les statistiques descriptives pour les tâches de mémoire de travail et de compétence langagière. Le Tableau 1 contient les valeurs moyennes et les écarts types des résultats obtenus par les participants aux tâches de mémoire de travail et de compétence langagière.

\section{Tableau 1}

Statistiques descriptives : mémoire de travail et compétence langagière

\begin{tabular}{ccc}
\hline & Moyenne & Écart type \\
\hline Mémoire de travail & 11,47 & $(1,92)$ \\
Compétence langagière & 11,90 & $(3,72)$ \\
\hline
\end{tabular}

Comme le montre le Tableau 1, les participants ont obtenu un résultat moyen de 11,9 $(\min .=5, \max .=18)$ que nous interprétons comme étant de niveau intermédiaire. On note également qu'ils ont obtenu un résultat moyen de 11,47 à la tâche de mémoire de travail (M $=11,47$; $\min .=7$, $\max .=15$ ).

Nous présentons dans le Tableau 2 la fréquence de production d'ARAA au total et pour chaque type, soit A-forme et A-choix, en fonction du nombre de mots produits pendant les deux minutes 34 secondes d'enregistrement transcrit. Nous présentons également les valeurs d'asymétrie ainsi que l'erreur type associés à chaque résultat.

Tableau 2

Statistiques descriptives : ARAA

\begin{tabular}{cccccccc}
\hline$n$ mots & ARAA & $\begin{array}{c}\text { Ratio } \\
\text { ARAA }\end{array}$ & A-forme & $\begin{array}{c}\text { Ratio } \\
\text { A- } \\
\text { forme }\end{array}$ & A-choix & $\begin{array}{c}\text { Ratio } \\
\text { A-choix }\end{array}$ \\
\hline Moyenne & 214,23 & 19,30 & 0,096 & 3,33 & 0,017 & 15,96 & 0,079 \\
& & & & & & & \\
Écart type & 63,85 & 8,80 & 0,048 & 2,38 & 0,014 & 8,02 & 0,043 \\
$\begin{array}{c}\text { Asymétrie } \\
(0,427)\end{array}$ & 0,576 & 0,137 & 0,430 & 0,394 & 0,985 & 0,293 & 0,505 \\
\hline
\end{tabular}

Note. $n=30 ;$ ARAA $=$ fréquence totale.

À la lecture du Tableau 2, il est possible de constater que les participants ont produit beaucoup plus d'ARAA de type A-choix, que de type A-forme. Afin de vérifier si cette différence est statistiquement significative, nous avons eu recours au test de Wilcoxon, nos données n'étant pas normalement distribuées. Cette différence s'est avérée significative ( $\mathrm{Z}$ $=-4,782 ; p=0,000)$.

Nous avons, ensuite, effectué une analyse factorielle en composantes principales incluant les ratios d'ARAA de type A-choix, les ratios d'ARAA de type A-forme, les 
résultats obtenus de la tâche de mémoire de travail, de la mesure de compétence langagière afin de vérifier la relation existant entre nos variables à l'étude.

Le Tableau 3 contient les valeurs propres, la variance expliquée par chacune des dimensions et le pourcentage de variance cumulée du modèle calculé. La représentation graphique du test d'accumulation de la variation permettant de déterminer le nombre de dimensions à extraire des données a révélé que deux dimensions existaient de manière statistiquement significative. Ces deux dimensions correspondent aux deux premiers axes présentés dans le Tableau 3.

\section{Tableau 3}

Résultats de l'analyse factorielle en composantes principales

\begin{tabular}{llll}
\hline Axe & Valeurs propres & $\begin{array}{l}\text { \% de la variance } \\
\text { expliquée }\end{array}$ & $\%$ cumulé \\
\hline 1 & 2,021 & 50,521 & 50,521 \\
2 & 1,051 & 25,259 & 75,780 \\
3 & 0,701 & 17,523 & 93,030 \\
4 & 0,268 & 6,698 & 100 \\
\hline
\end{tabular}

Selon les données présentées dans le Tableau 3, les deux premières dimensions rendent compte de près de $76 \%$ de la variation. La Figure 1 présente les dimensions en fonction de leurs valeurs propres.

\section{Figure 1}

Projection des variables sur l'espace bidimensionnel des deux dimensions

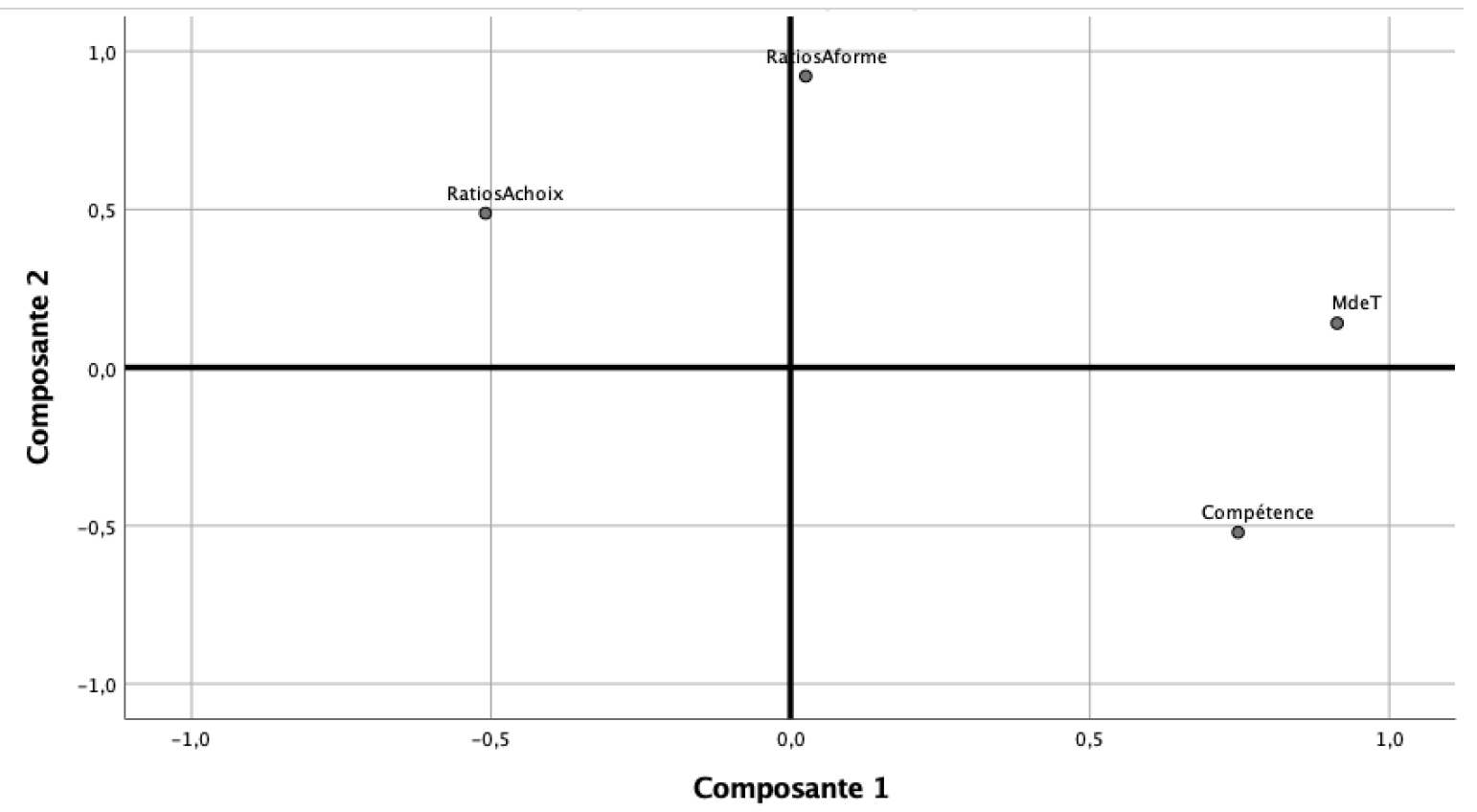

Il est possible d'observer à la Figure 1, qu'à l'horizontale, une dimension se crée sur l'opposition des ratios d'ARAA de type A-choix (corrélation dimension-variable = -0,51) à 
la mesure de mémoire de travail (corrélation dimension-variable $=0,91$ ) et à la mesure de compétence langagière (corrélation dimension-variable $=0,75$ ). Cette première dimension, qui explique $50 \%$ de la variation observée, révèle qu'une meilleure mémoire de travail est corrélée avec une production moindre d'ARAA visant un choix de mots ou de groupes de mots et un résultat plus élevé à la tâche de compétence langagière. À la verticale, on observe une seconde dimension constituée des ratios d'ARAA de type A-forme (corrélation dimension-variable $=0,92$ ) et qui est associée de façon négative à la mesure de compétence orale (corrélation dimension-variable $=-0,52$ ), indiquant qu'un plus grand nombre d'ARAA visant la forme est corrélé négativement avec le niveau de compétence langagière.

\section{Discussion}

Nous nous étions donné comme objectif d'examiner les liens existant entre l'aspect exécutif de la mémoire de travail, mesuré au moyen d'une tâche numérique et la production d'ARAA en français L2 en répondant à la question suivante : quelle est la relation entre l'aspect exécutif de la mémoire de travail mesurée à l'aide de la tâche du nombre le plus grand (Oakhill et coll., 2011) et la production d'ARAA réalisées lors d'une tâche de narration à partir d'images ?

Pour ce faire, 30 adultes sinophones apprenants du français ont réalisé la tâche du nombre le plus grand et une narration à partir d'images. Dans ce qui suit, nous présentons, d'abord, une discussion générale de nos résultats au regard des travaux antérieurs sur les ARAA et passons ensuite à une discussion au regard de notre question de recherche.

De manière générale, les ratios d'ARAA calculés ont révélé qu'un plus grand nombre d'ARAA de type A-choix avaient été produites par nos participants. Cette différence observée entre les ratios d'ARAA de type A-choix et ceux de type A-forme s'est révélée être statistiquement significative. Ce comportement est similaire à celui observé dans les études antérieures qui mettent en lumière des différences entre les catégories d'ARAA (p. ex., Arroyo, 2003; Griggs, 2002; Kormos, 2000a, 2000b; Mojavezi et Ahmadian, 2013; O'Connor, 1988; Simard et coll., 2011, 2019; Verhoeven, 1989; Zuniga, 2015). De la même manière, ces résultats sont similaires à ceux obtenus par Simard et ses collaborateurs (2016) qui ont également observé une plus grande production d'ARAA de type A-choix chez leurs participants francophones apprenants de l'anglais L2. Il est intéressant de préciser ici que les participants de la présente étude ont obtenu un résultat similaire à ceux de l'étude antérieure pour la compétence langagière, soit de 11,90 sur 18 pour nos participants et de 12,16 également sur 18 pour Simard et ses collaborateurs (2016) (la même grille d'évaluation de la compétence langagière a été utilisée dans les deux études). Notre résultat corrobore donc ceux obtenus d'études antérieures qui montrent que plus les apprenants sont avancés, plus ils formulent des ARAA de type A-choix (p. ex., Kormos, 2000a, 2000b; O'Connor, 1988; Verhoeven, 1989; Zuniga, 2015; Zuniga et Simard, 2019).

Afin d'atteindre notre objectif de recherche et de répondre à notre question de recherche, nous avons effectué une analyse factorielle en composantes principales. Les résultats de cette analyse mettant en relation la mémoire de travail, la compétence langagière et les ARAA de types A-choix et A-forme, ont révélé que les ARAA de type Achoix étaient négativement associées à la mémoire de travail. Ainsi, meilleur est l'aspect exécutif de la mémoire de travail, moins nombreuses sont les ARAA visant les choix de mots ou de groupes de mots dans le discours. Il est possible de penser qu'une meilleure 
capacité de mémoire de travail permet une plus grande efficacité de récupération des mots ou de groupes de mots (p. ex., Baddeley, 2007), ce qui mènerait à la diminution d'ARAA de type A-choix post-verbalement. Par ailleurs, les résultats de l'analyse factorielle mettent également en lumière qu'une plus grande production d'ARAA de type A-forme est associée exclusivement à une moins bonne compétence langagière.

Aussi, il est intéressant de noter que les ARAA visant le choix et celles visant la forme sont représentées de façon orthogonale sur le plan cartésien dans lequel se trouvent les deux facteurs issus de l'analyse factorielle, appuyant ainsi l'idée selon laquelle les deux types d'ARAA qui ont été codés dans notre étude sont produits lors de deux phases différentes dans le processus de production de la parole, tel que conçu par Levelt (1989, 1999), soit respectivement la phase de la conceptualisation et la phase de la formulation (Zuniga, 2015; Zuniga et Simard, 2019). Rappelons ici que nous avons codé les ARAA selon qu'elles visent le contenu du discours, que nous associons à la phase de la conceptualisation, ou encore la forme de la langue, que nous associons à la phase de la formulation.

Cela dit, même si notre codification des ARAA diffère quelque peu des types retenus dans les études de Ahmadian (2015) et de Mojavezi et Ahmadian (2013), nos résultats sont tout de même similaires aux leurs. En effet, ils ont tout comme nous observé une association négative entre les ARAA visant un changement dans le discours et la mémoire de travail. Toutefois, rappelons que Georgiadou et Roehr-Brackin (2017) n'ont pour leur part observé aucune relation entre leurs mesures de l'aspect exécutif de la mémoire de travail et la production d'ARAA de leurs participants, peu importe le type. D'une part, il est important de préciser que les auteures ont passé leur tâche d'empan d'écoute dans la L2 de leurs participantes. Il est donc possible de penser qu'un chevauchement partiel des contenus examinés a masqué des effets possibles. D'autre part, les auteures ont effectué leurs analyses sur le nombre brut d'ARAA, dans les deux minutes d'entretien sélectionné, sans effectuer de transformation en ratio, soit le nombre brut d'ARAA divisé par le nombre de mots produits dans les deux minutes de transcription (voir Biber et coll., 2006). Comme certains locuteurs produisent plus de mots par minute que d'autres, ils ont par conséquent plus de chance de produire d'ARAA simplement à cause de leur débit de parole. Il est essentiel d'avoir une mesure de base comparable des comportements d'ARAA. Donc, les résultats des analyses effectuées par les deux auteures ne permettent peut-être pas de mettre en relief les relations qui existent entre l'aspect exécutif de la mémoire de travail et les ARAA.

Mentionnons au passage que dans le cadre d'une étude antérieure sur la relation entre la mémoire phonologique et la production d'ARAA en anglais L2 (Simard et coll., 2016), les résultats des analyses factorielles en composantes principales avaient révélé qu'une meilleure mémoire phonologique était en lien avec une production moindre d'ARAA centrées sur la forme. Rappelons que dans le cadre de la présente étude, une meilleure mémoire de travail (aspect exécutif) est associée avec une production moindre d'ARAA centrées sur le choix de mot ou de groupes de mots. Ces résultats laissent entendre que les variables cognitives interagissent de façon différenciée avec les différents types d'ARAA. Il serait intéressant de vérifier au sein de la même étude, de quelle manière les ressources cognitives sont sollicitées selon les différents types d'ARAA afin d'offrir des informations additionnelles aux connaissances actuelles et ainsi de pouvoir préciser le portrait existant relativement à la relation entre les ressources cognitives et la production d'ARAA. 
Par ailleurs, bien que nos résultats fournissent des informations additionnelles quant à la relation entre une variable individuelle cognitive, nommément l'aspect exécutif de la mémoire de travail et la production d'ARAA, d'autres facteurs interagissent certainement avec ces deux variables. Nous pensons notamment à l'effet du type de tâches. Rappelons que Georgiadou et Roehr-Brackin (2017) ont utilisé une tâche d'entrevue, que les deux études antérieures ont quant à elles eu recours à une tâche de narration à partir d'un film muet et que dans notre étude nous avons eu recours à une narration à partir d'images. Il serait donc intéressant de vérifier de quelle manière interagissent les ressources cognitives, le type de tâches et la production d'ARAA. Nous pensons également à l'effet de différentes conditions d'exécution des tâches de production orale (p. ex., narration planifiée ou non planifiée). Bien qu'Ahmadian (2015) ait vérifié la relation entre la planification en temps réel (sans temps préalable de préparation et avec aucune restriction pour la réalisation de la narration), il n'a toutefois pas comparé les résultats obtenus de cette condition expérimentale à ceux de groupes témoins. Il serait donc intéressant d'analyser l'interaction entre les aspects exécutifs de la mémoire de travail, les différentes conditions de l'exécution d'une tâche de production orale (p. ex., sans planification préalable, et avec restriction de temps pour la réalisation de la narration; avec planification préalable et sans restriction de temps pour la réalisation de la narration, etc.) et la production d'ARAA.

\section{Conclusion}

La production orale constitue un défi de taille pour les locuteurs non natifs. Il est donc primordial de bien en comprendre tous les rouages afin d'être mieux à même d'aider éventuellement les apprenants de L2. À cet égard, les conclusions de notre étude nous informent quant à l'association entre l'aspect exécutif de la mémoire de travail et les modifications visant le discours. Ainsi, meilleure est la capacité de mémoire de travail, moins nombreuses sont les interventions sur les choix de mots ou de groupes de mots. Évidemment, davantage d'études sont nécessaires afin d'obtenir un portrait complet des relations entre la production d'ARAA et les variables cognitives. À cet égard, nous espérons avoir ouvert la discussion vers de nouvelles idées de recherche.

La correspondance devrait être adressée à Daphnée Simard.

Courriel : $\underline{\text { simard.daphnee@,uqam.ca }}$

\section{Remerciements}

Nous tenons à remercier sincèrement les évaluateurs anonymes de la Revue canadienne de linguistique appliquée, qui ont contribué à l'amélioration de la qualité de notre article. Nous tenons également à remercier chaleureusement nos participants sans qui cette étude n'aurait pas été possible.

\section{Notes}

${ }^{1}$ Par ailleurs, si certaines recherches ont porté sur les ARAA masquées (covert) (voir Levelt, 1983) - qui se présentent entre autres sous la forme de faux départs et d'hésitations ce sont principalement les ARAA manifestes (overt), étant donnée leur nature concrète, qui ont été l'objet d'un plus grand nombre de recherches (Simard et coll., 2011). 
${ }^{2}$ La mémoire de travail et la mémoire phonologique sont traitées comme étant des construits indépendants l'un de l'autre, car les résultats des travaux mettent en évidence leurs contributions significatives distinctes dans différents phénomènes liés à l'acquisition des langues secondes (Wen, 2019), que ce soit dans le développement du vocabulaire que de la grammaire pour ne nommer qu'eux (p. ex., Martin et Ellis, 2012).

${ }^{3}$ La tâche d'empan d'écoute constitue une variation de la tâche d'empan de lecture, développée à l'origine par Daneman et Carpenter (1980), et est de loin la plus utilisée afin de mesurer l'aspect exécutif de la mémoire de travail (voir Wen, 2016 pour une synthèse).

${ }^{4}$ Lors d'une tâche de rappel suscité, les participants sont invités à commenter leurs propres réalisations à la suite de la passation d'une tâche langagière (Gass, 2000).

${ }^{5}$ Bien que les chercheurs fassent une distinction entre les tâches verbales et numériques, elles impliquent néanmoins l'emmagasinage temporaire de matériel verbal (nombres, mots), au moyen de la composante boucle phonologique de la mémoire de travail. Ainsi seule la composante de traitement de l'information de la tâche s'avère réellement non verbale (traitement numérique), dans le cas des tâches numériques (voir Oakhill et coll., 2011)

${ }^{6}$ Lorsque ces tâches numériques sont utilisées dans des études en L2, il est recommandé de les passer dans la L1 des participants afin de réduire le biais potentiel de connaissance de la langue (avec une meilleure connaissance de la langue associée à de meilleurs résultats) dans la composante emmagasinage de la tâche (voir French, 2006, pour plus de détails).

\section{Références}

Ahmadian, M. J. (2015). Working memory, online planning and L2 self-repair behaviour. Dans Z. Wen, M. B. Mota et A. McNeill (dir.), Working memory in second language acquisition and processing (pp. 160-174). Multilingual Matters.

Arroyo, E. (2003). La reformulation en communication exolingue chez des locuteurs hispanophones parlant français. Marges linguistiques, septembre-octobre. http://www.marges-linguistiques.com

Baddeley, A. D. (1986). Working memory. Oxford University Press.

Baddeley, A. D. (1992). Working memory. Science, 255, 556-559.

Baddeley, A. D. (2000). The episodic buffer: A new component of working memory?

Trends in Cognitive Science, 4, 417-423.

Baddeley, A. D. (2007). Working memory, thought and action. Oxford University Press.

Baddeley, A.D. (2010). Working memory. Current Biology, 20, 136-140.

Baddeley, A. D. (2012). Working memories: Theories, models, and controversies. Annual Review of Psychology, 63, 1-29.

Baddeley, A. D., Gathercole, S. et Papagano, C. (1998). The phonological loop as a language learning device. Psychological Review, 105, 158-173. 
Baddeley, A. D. et Hitch, G. J. (1974). Working memory. Dans G.H. Bower (dir.), The psychology of learning and motivation: Advance in research and theory (pp. 47-89). Academic Press.

Bange, P. et Kern, S. (1996). La régulation du discours en L1 et en L2. Études romanes, 35, 69-103.

Biber, D., Conrad, S. et Reppen, R. (2006). Corpus linguistics: Investigating language structure and use ( $5^{\text {th }} \mathrm{ed}$.). Cambridge University Press.

Camps, J. (2003). The analysis of oral self-correction as a window into the development of past time reference in Spanish. Foreign Language Annals, 36, 233-242.

Cattell, R. B. (1966). The scree test for the number of factors. Multivariate Behavioural Research, 1, 245-276.

Colom, R., Abad, F. J., Rebello, I. et Shih, P. C. (2005). Memory span and general intelligence: A latent variable approach. Intelligence, 33, 623-642.

de Bot, K. (1992). A bilingual production model: Levelt's "speaking" model adapted. Applied Linguistics, 13, 1-24.

Fathman, A. (1980). Repetition and correction as an indication of speech planning and execution processes among second language learners. Dans H. Dechert et M. Raupach (dir.), Toward a cross-linguistic assessment of speech production (pp. 7785). Verlag Peter D. Lang.

Fincher, A. (2006). Functions of self-initiated self-repairs in an advanced Japanese language classroom. [thèse de doctorat inédite]. Griffith University, Brisbane, Australia.

Foster, P. et Skehan, P. (1996). The influence of planning and task type on second language performance. Studies in Second Language Acquisition, 18, 299-323.

French, L. (2006). Phonological working memory and second language acquisition. A developmental study of francophone children learning English in Quebec. The Edwin Mellen Press.

Gass, S. (2000). Stimulated recall methodology in second language research. Routledge.

Georgiadou, E. et Roehr-Brackin, K. (2017). Investigating executive working memory and phonological short-term memory in relation to fluency and self-repair behaviour in L2 speech. Journal of Psycholinguistic Research, 46, 877-895.

Gilabert, R. (2007). Effects of manipulating task complexity on self-repairs during L2 oral production. International Review of Applied Linguistics, 45, 215-240.

Griggs, P. (1997). Metalinguistic work and the development of language use in communicative pair-work activities involving second language learners. Dans L. Diaz et C. Pérez (dir.), Views on the acquisition and the use of second languages (pp. 403-415). Presses de l'Universitat Pompeu Fabrat.

Griggs, P. (1998). L'effet de la tâche sur la régulation de l'activité métalinguistique entre apprenants partageant la même langue maternelle. Dans M. Souchon (dir.), Pratiques discursives et acquisition des langues étrangères (pp. 103-115). Presses de l'Université de Franche-Compté.

Griggs, P. (2002). À propos de l'effet de l'activité métalinguistique sur les processus de production en L2. Dans D. Véronique et F. Cicurel (dir.), Discours, action et appropriation des langues (pp. 53-66). Presses Sorbonne Nouvelle.

Griggs, P. (2007). Perspective sociocognitive sur l'apprentissage des langues étrangères: situation naturelle ou guidée. L'Harmattan. 
Harrington, M. et Sawyer, M. (1992). L2 working memory capacity and L2 reading skill. Studies in Second Language Acquisition, 14, 25-38.

Hergé (1931). Tintin au Congo. Casterman.

Kormos, J. (1999a). Monitoring and self-repair in L2. Language Learning, 49, 303-342.

Kormos, J. (1999b). The effect of speaker variables on the self-correction behaviour of L2 learners. System, 27, 207-221.

Kormos, J. (2000a). The role of attention in monitoring second language speech production. Language Learning, 50, 343-384.

Kormos, J. (2000b). The timing of self-repairs in second language speech production. Studies in Second Language Acquisition, 22, 145-167.

Kormos, J. (2006). Speech production and second language acquisition. Lawrence Erlbaum Associates.

Larson-Hall, J. (2010). A guide to doing statistics in second language research using SPSS. Routledge.

Lennon, P. (1984). Retelling a story in English as a second language. Dans H. W. Dechert, D. Mohle et M. Raupach (Dir.), Second language productions (pp. 50-68). Gunter Narr.

Lennon, P. (1990). Investigating fluency in EFL: A quantitative approach. Language Learning, 40, 387-417.

Levelt, W. J. M. (1983). Monitoring and self-repair in speech. Cognition, 33, 41-103.

Levelt, W. J. M. (1989). Speaking: From intention to articulation. MIT Press.

Levelt, W. J. M. (1999). Language production: A blueprint of the speaker. In C. Brown et P. Hagoort (ed.), Neurocognition of language (pp. 83-122). Oxford University Press.

Martin, K. I. et Ellis, N. C. (2012). The roles of phonological STM and working memory in L2 grammar and vocabulary learning. Studies in Second Language Acquisition, 34, $379-413$.

Mehnert, U. (1998). The effect of different lengths of time for planning in second-language performance. Studies in Second Language Acquisition, 20, 83-108.

Miyake, A. et Friedman, N. P. (1998). Individual differences in second language proficiency: Working memory as language aptitude. Dans A. F. Healy et L. E. Bourne (ed.), Foreign language learning: Psycholinguistic studies on training and retention (pp. 339-364). Laurence Erlbaum Asscociates.

Mojavezi, A. et Ahmadian, M. J. (2013). Working memory capacity and self-repair behaviour in first and second language oral production. Journal of Psycholinguistic Research, 43, 289-297.

Munro, M. J. et Derwing. T. M. (1995). Foreign accent, comprehensibility, and intelligibility in the speech of second language learners. Language Learning, 45, 73 97.

Oakhill, J., Yuill, N. et Garnham, A. (2011). The differential relations between verbal, numerical and spatial working memory abilities and children's reading comprehension. International Electronic Journal of Elementary Education, 4, 83106.

O’Brien, I., Segalowitz, N., Freed, B. et Collentine, J. (2007). Phonological memory predicts second language oral fluency gains in adults. Studies in Second Language Acquisition, 29, 557-581. 
O’Connor, N. (1988). Repairs as indicative of interlanguage variation and change. Dans T. J. Walsh (dir.), Georgetown University round table in languages and linguistics 1988: Synchronic and diachronic approaches to linguistic variation and change (pp. 251-259). Georgetown University Press.

Poulisse, N. (1997). Language production in bilinguals. Dans A. de Grot et J. Kroll (dir.), Tutorials in bilingualism: Psycholinguistic perspectives (pp. 201-224). Lawrence Erlbaum Associates.

Rosen, V. M. et Engle, R. W. (1997). Forward and backwards serial recall. Intelligence, 25, 37-47.

Rossiter, J. M., Derwing, T. M. et Jones, V. L. M. O. (2008). Is a picture worth a thousand words? TESOL Quarterly, 42, 325-329.

Royer, C. (2002). L'appropriation de la négation du français en milieu guidé: une approche interactionniste. Marges linguistiques, novembre. http://www.margeslinguistiques.com.

Salonen, T. et Laakso, M. (2009). Self-repair of speech by four-year-old Finnish children. Journal of Child Language, 36, 855-882.

Schegloff, E.A., Jefferson, G. et Sacks, H. (1977). The preference for self-correction in the organization of repair in conversation. Language, 53, 361-382.

Segalowitz, N. (2010). Cognitive bases of second language fluency. Routledge.

Simard, D., Bergeron, A., Liu, Y.-G., Nader, M., et Redmond, L. (2016). Production d'autoreformulations autoamorcées en langue seconde : rôle de l'attention et de la mémoire phonologique. Canadian Modern Language Review/La Revue canadienne des langues vivantes, 72, 183-210.

Simard, D., Fortier, V., et Zuniga, M. (2011). Attention et production d'autoreformulations autoamorcées en français langue seconde, quelle relation? Journal of French Language Studies, 21, 3, 417-436.

Stafford, J. et Bodson, P. (2006). L'analyse multivariée avec SPSS. Presses de 1'Université du Québec.

St Clair-Thompson, H. L. (2010). Backwards digit recall: A measure of short-term memory or working memory? European Journal of Cognitive Psychology, 22, 286-296.

Tavakoli, P. et Foster, P. (2008). Task design and second language performance: The effect of narrative type on learner output. Language Learning, 58, 439-473.

Turner, M. L. et Engle, R. W. (1989). Is working memory capacity task dependent? Journal of Memory and Language, 28, 127-154.

Turner, C. E. et Upshur, J. (2002). Rating scales derived from student samples: Effects of the scale maker and the student sample on scale content and student scores. TESOL Quarterly, 36, 49-70.

Upshur, J. A. et Turner, C. E. (1995). Constructing rating scales for second language tests. English Language Teaching Journal, 49, 3-12.

Upshur, J. A. et Turner, C. E. (1999). Systematic effects in the rating of second language speaking ability, Test method and learner discourse. Language Testing, 16, 82-111.

Van der Linden, M. et Collette, F. (2002). Attention et mémoire de travail. Dans J. Couillet, M. Leclercq, C. Moroni et P. Azouvi (dir.), La neuropsychologie de l'attention (pp. 41-54). Solale.

Verhoeven, L. T. (1989). Monitoring in children's second language speech. Second Language Research, 5, 141-155. 
White, J. et Turner, C. E. (2005). Comparing children's oral ability in two ESL programs. Canadian Modern Language Review/La revue canadienne des langues vivantes, 61, 491-517.

Yuan, F. et Ellis, R. (2003). The effects of pre-task planning and on-line planning on fluency, complexity and accuracy in L2 monologic oral production. Applied Linguistics, 24, 1-27.

Zuniga, M. (2015). Attention et production orale en langue seconde: la relation entre attention et production d'autoreformulations autoamorcées. [thèse de doctorat inédite]. Université du Québec à Montréal, Montréal, Canada.

Zuniga, M., et Simard, D. (2019). Factors influencing L2 self-repair behaviour: The role of L2 proficiency, attentional control and L1. Journal of Psycholinguistic Research, 48, 43-59.

Wen, Z. (2016). Phonological and executive working memory in L2 task-based speech planning and performance. The Language Learning Journal, 44, 418-435.

Wen, Z. (2019). Working memory as language aptitude. Dans Z. Wen, P. Skehan, A. Biedron, S. Li, et R. Sparks (dir.), Language aptitude: Advancing theory, testing, research, and practice (pp. 1-23). Routledge. 